\title{
A Semi Blind Self Reference Image Watermarking in Discrete Cosine Transform using Singular Value Decomposition
}

\author{
Satyanarayana Murty. P \\ Research Scholar \\ Dept of ECE \\ $\mathrm{AU}$ college of Engineering (A) \\ Andhra University \\ Vishakhapatnam
}

\author{
Sekher Dileep Kumar \\ Assistant Professor \\ Dept of ECE \\ GIITS, COE.
}

\author{
Rajesh Kumar. P \\ Associate Professor \\ Dept of ECE \\ AU college of Engineering $(A)$ \\ Andhra University \\ Vishakhapatnam
}

\begin{abstract}
An approach to self reference image watermarking scheme based on Discrete Cosine Transform (DCT) and Singular Value Decomposition (SVD). First, the original image is segmented into blocks by using ZIG - ZAG sequence. Then find the edges in each block and kept a threshold on these number of edges in each block. Using this threshold, get the essential blocks. A reference image is formed by taking these essential blocks. Reference image is transformed into DCT domain and then SVD is performed on both reference and watermarked image. Embedding is done by modifying singular values of the reference image using singular values of watermark. After embedding, modified reference image is segmented into blocks and these modified blocks are mapped into their original places for constructing watermarked image. For extraction, the watermarked reference image is constructed by the watermarked image using the positions of the selected blocks and the reverse process is used for extracting the singular values of the watermark from watermarked reference image. Robustness of proposed scheme is carried out by a variety of attacks.
\end{abstract}

\section{Keywords}

DCT, SVD, Zig-Zag, Robust, edges

\section{INTRODUCTION}

The success of the Internet, cost-effective and popular digital recording and storage devices, and the promise of higher bandwidth and quality of service (QoS) for both wired and wireless networks has made it possible to create, replicate, transmit, and distribute digital content in an effortless way. So the protection of digital multimedia content has become an increasingly important issue for content owners and service providers. As watermarking is identified as a major technology to achieve copyright protection, the relevant literature [1-6] includes several distinct approaches for embedding data into a multimedia element. The major applications of digital watermarking were copyright protection, authentication, fingerprinting, broad cast monitoring etc, [7-12]. Watermarking can be implemented in spatial domain, frequency domain and hybrid domain. The simple technique in spatial domain was Least Significant Bit (LSB) technique [13, 14]. Transform domain watermarking is useful for taking advantage of perceptual criteria in the embedding process, for designing watermarking techniques which are robust to common compression techniques, and for direct watermark embedding of compressed bit streams. A common transform framework for images is the block-based DCT which is a fundamental building block of current image coding standards such as JPEG and video coding standards such as the MPEG video coders. The DCT is performed on $8 \times 8$ blocks of data, pseudorandom subsets of the blocks are chosen and a triplet of midrange frequencies is slightly altered to encode a binary sequence. This is a reasonable heuristic watermarking approach since watermarks inserted in the high frequencies are vulnerable to attack whereas the low frequency components are perceptually significant and sensitive to alterations. A DCT is performed on the whole image and the watermark is inserted in a predetermined range of low frequency components minus the DC component. The watermark consists of a sequence of real numbers generated from a Gaussian distribution which is added to the DCTcoefficients. The watermark signal is scaled according to the signal strength of the particular frequency component. This is a reasonable and simple way to introduce some type of perceptual weighting into the watermarking scheme [15-29]. Singular Value Decomposition (SVD) [30-36] is available in the literature. The basic philosophy in majority of the transform domain watermarking schemes is to modify transform coefficients based on the bits in watermark image.

A semi-blind digital watermarking scheme based on DCT-SVD is proposed. The rest of the paper is organized as follows: Section 2 describes related work, in section 3 the proposed watermarking method, section 4 contains our experimental results followed by conclusions in Section 5 and references in section 6 .

\section{RELATED WORK}

Alexander Sverdlov et al [37], proposed a new robust hybrid watermarking scheme based on DCT and SVD. After applying the DCT to the cover image, they map the DCT coefficients in a zig-zag order into four quadrants, and apply the SVD to each quadrant. These four quadrants represent frequency bands from the lowest to the highest. The singular values in each quadrant are then modified by the singular values of the DCT-transformed visual watermark. They assume that the size of the visual watermark is one quarter of the size of the cover image. They show that embedding data in lowest frequencies is resilient to one set of attacks while embedding data in highest frequencies is resilient to another set of attacks. They compare their hybrid algorithm with a pure SVD-based scheme.

Liu Quan, and AI Qingsong [38], proposed a new watermarking algorithm which combines both merits of the algorithm based on discrete cosine transform (DCT) and the algorithm based on singular value decomposition. 
Fangjun Huang and Zhi-Hong Guan [39], a new hybrid SVDDCT watermarking method is presented. The SVD transform and the DCT are performed on the watermark and the original image, respectively. Only the SVs of the watermark are embedded into the DCT coefficients of original image. For convenience, they divide the SVs into two groups. The group including the bigger elements is named Group A, and the group including the remaining ones is named Group B. The elements in Group A which comprise most energy of the watermark are embedded into the DC components of the subblocks of the original image. The elements in Group B are embedded into the remaining significant DCT components.

Feng Liu, and Yangguang Liu [40], proposed a watermarking algorithm for digital image based on DCT and SVD. This algorithm demonstrates that the watermarking is robust to the common signal processing techniques including JEPG compressing, noise, low pass filter, median filter, contrast enhance. They compare their work with the existing SVD methods.

\section{PROPOSED ALGORITHM}

A semi-blind watermarking scheme using DCT and SVD is proposed. The host and watermark images are gray scale images of size $\mathrm{M} \times \mathrm{N}$ and $\mathrm{m} \times \mathrm{n}$ respectively, which are denoted by $\mathrm{F}$ and $\mathrm{W}$. Block diagram of proposed watermarking scheme is shown in figure 1

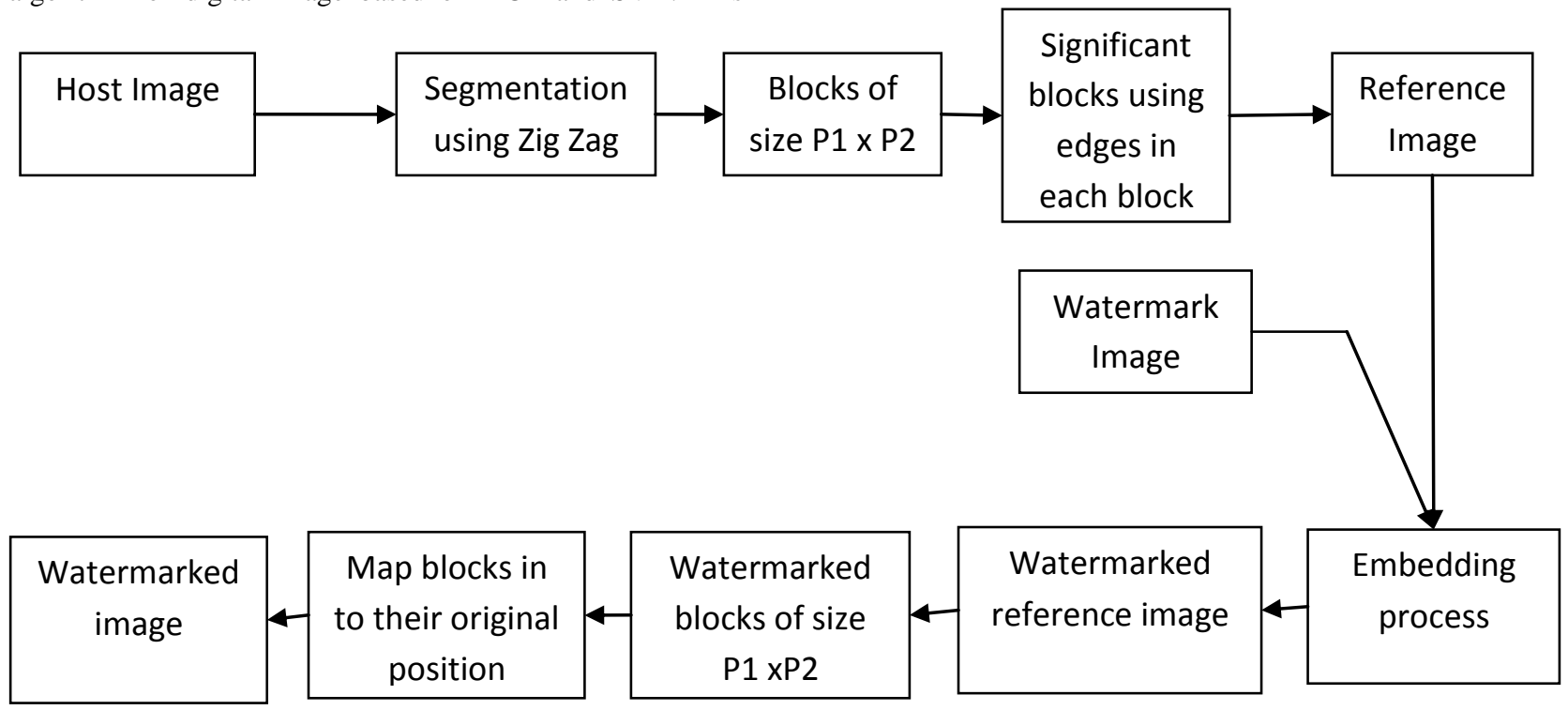

Figure 1: Embedding process

\subsection{Watermark embedding procedure}

First, the original image is segmented into blocks of size $\mathrm{p}_{1} \times \mathrm{p}_{2}$ via ZIG_ZAG sequence denoted by $\mathrm{F}^{\mathrm{l}}$, where 1 is the number of blocks.

Step1: Find out the number of edges in each block.

Step2: The numbers of edges in each block are stored in descending order. Then make a threshold on the number of edges in each block. Those blocks, which have number of edges less than or equal to threshold, are considered as significant blocks and are used for making reference image, $F_{\text {ref }}$ which is a size of $\mathrm{m} \times \mathrm{n}$.

Step3: Perform DCT on the reference image, which is denoted by $f_{D C T}$

Step4: Perform SVD on $f_{D C T}$, which is denoted by

$f_{D C T}^{S V D}=U_{D C T} * S_{D C T} * V_{D C T}^{T}$

\subsection{Watermark extraction procedure}

Watermark extraction procedure as follows:
Step4: Perform SVD transform on watermark image W, which is denoted by

$$
f_{W}^{S V D}=U_{W} * S_{W} * V_{W}^{T}
$$

Step5: Modify the singular values of reference image with the singular values of watermark as

$$
\left(S_{\text {ref }}\right)^{*}=S_{D C T}+\beta * S_{w}
$$

Where, $\beta$ gives the watermark depth.

Step7: Perform inverse SVD,

$$
f_{i s v d}^{*}=U_{D C T} * S_{r e f}^{*} * V_{D C T}^{T}
$$

Step8: Perform inverse DCT to construct the modified reference image, denoted by. $F_{\text {ref }}^{*}$. Again $F_{\text {ref }}^{*}$ is segmented into blocks of size $\mathrm{p}_{1} \times \mathrm{p}_{2}$ and mapped onto

their original positions for constructing the watermarked image.

We save the positions of the significant blocks and reference image for the extraction process.

Step1: Using the positions of significant blocks, make the reference image from the watermarked image, denoted by $F_{W}^{r e f}$ 
Step2: Perform DCT on reference image, which is denoted by $f_{D C T}^{\text {Yef }}$

Step3: Perform SVD transform on $f_{D C T}^{\text {ref }}$.

$f_{\text {SVD }}^{\text {ref }}=U_{\text {ref }} * S_{\text {ref }} * V_{\text {ref }}^{T}$

Step4: Extract the singular values of the watermark.

$S_{W}^{\text {ext }}=\frac{S_{\mathrm{ref}}-S_{W}}{\beta}$

Step5: Obtain the extracted watermark as

$W^{\text {ext }}=U_{W} * S_{W}^{\mathrm{Ext}} * V_{W}^{T}$

\section{EXPERIMENTAL RESULTS}

The algorithm discussed in the above section has been implemented in MATLAB for the gray scale Boat image of size $512 \times 512$. For watermark, Lena gray scale image of size $256 \times 256$ is used. Both have showed in figure 2 .

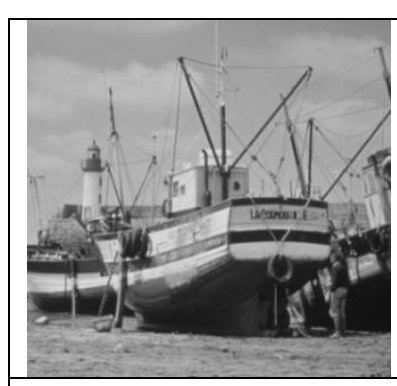

(a)

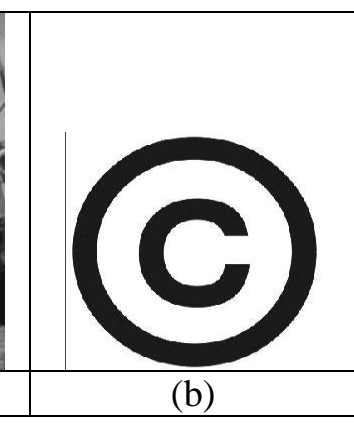

(b)
Figure 2: (a) Original Image (b) Watermark Image

In this experiment, the size of blocks is taken to be $8 \times 8$. It is investigated the robustness of the algorithm by considering Average filtering, Median filtering, Compression, Cropping, Gaussian noise, Histogram Equalization, Resize, Rotate, contrast adjustment, wrapping and Sharpening attacks.

Imperceptibility means that the perceived quality of the image should not be distorted by the presence of the watermark. The peak signal to noise ratio (PSNR) is typically used to measure the degradation between original image and watermarked image.

$$
\begin{aligned}
& R M S E=\sqrt{\frac{[f(i, j)-F(i, j)]^{2}}{N^{2}}} \\
& P S N R=20 \log \frac{255}{R M S E} d b
\end{aligned}
$$

RMS is the Root Mean Square Error and is a comparison between the host image and watermarked image. $f(i, j)$ and $F(i, j)$ represent host and watermarked images respectively. Size of the host image is $\mathrm{N} \times \mathrm{N}$.

Watermarked Boat image has the PSNR value of $48.68 \mathrm{db}$. If it observed that the original and watermarked images couldn't find any perceptual degradation. Watermarked boat image and extracted watermark are shown in figure 3.

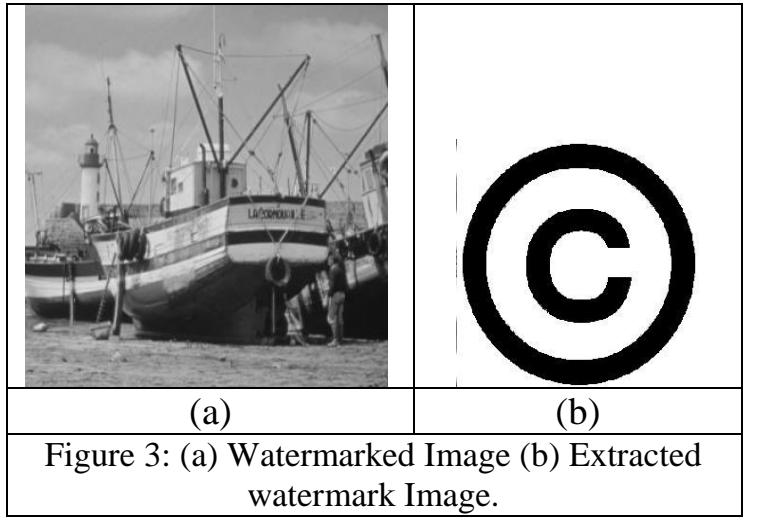

Robustness of a watermarking algorithm is that the embedded data should survive any signal processing operation. The similarity between the original watermark and the extracted watermark from the attacked watermarked image was measured by using the correlation factor $\rho$, which is computed using the following Equation:

$\rho(w, \widetilde{w})=\frac{\sum_{i=1}^{N} w_{i} \widetilde{w}_{i}}{\sqrt{\sum_{i=1}^{N} w_{i}^{2}} \sqrt{\sum_{i=1}^{N} \widetilde{w}_{i}^{2}}}$

Where $\mathrm{N}$ is the number of pixels in watermark, $\mathrm{w}$ and $\widetilde{w}$ is the original and extracted watermarks respectively. The correlation factor $\rho$, may take values between -1 and 1 .

The most common manipulation in digital image is filtering. In this work two types of filtering attacks have been considered. Average Filtering attack on watermarked image and extracted watermark after applying $13 \times 13$ averaging filtering are shown in fig 4(a) and fig 4(b) respectively. In fig 5(a) and fig 5(b) shows the median Filtering attack on watermarked image and extracted watermark after applying 13 x 13 median filtering.

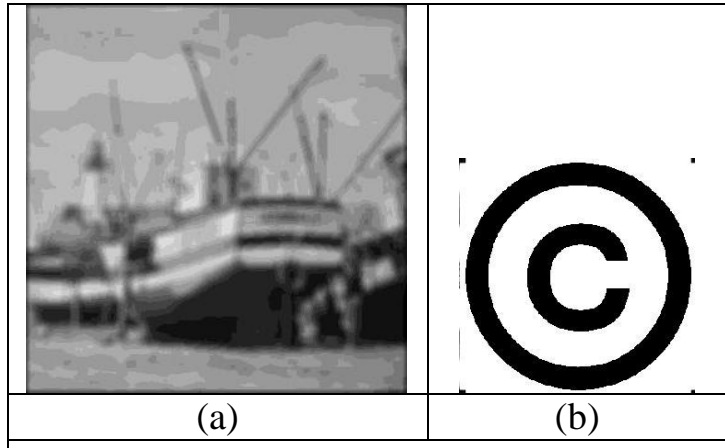

Figure 4: Results of average Filtering attack $(13 \times 13)$ 


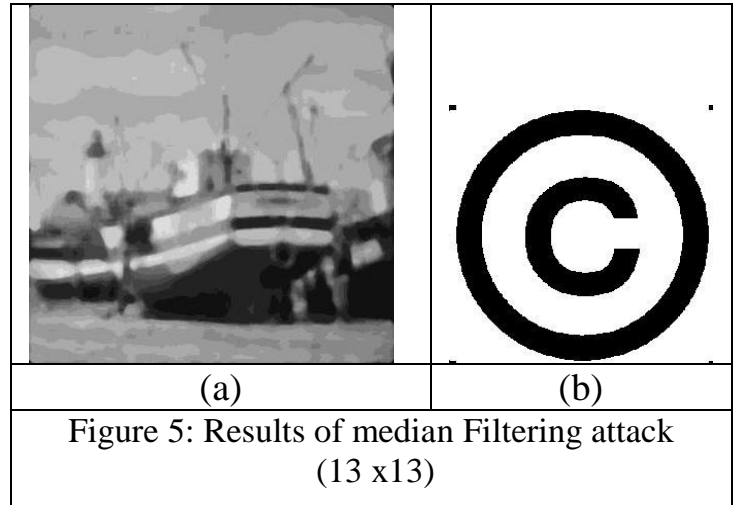

To verify the robustness of the watermarking scheme, another measure is noise addition. In real life, the degradation and distortion of the image come from noise addition. In this experiment, it is added $75 \%$ additive Gaussian noise on the watermarked image. In fig 6(a) was the median filtering attack on watermarked image and fig 6(b) was extracted watermark.

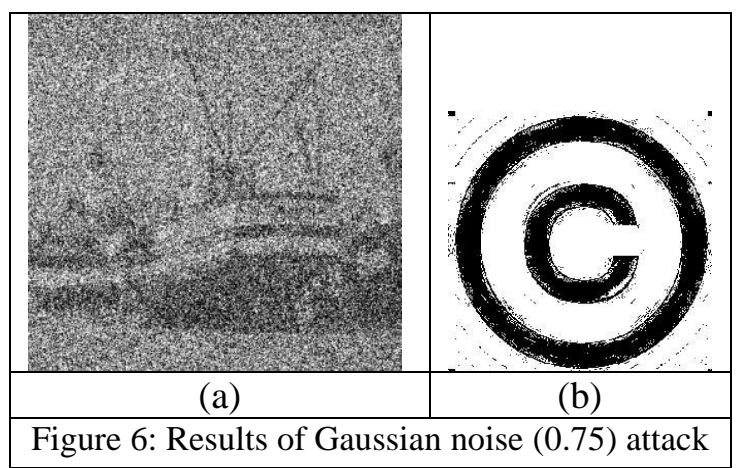

In real life applications, storage and transmission of digital data, a lossy coding operation is often performed on the data to reduce the memory and increase efficiency. Hence, this algorithm was tested for the JPEG compression (80:1) and the tested results are shown in figure 7(a) compression attacked image and fig 7(b) extracted watermark.

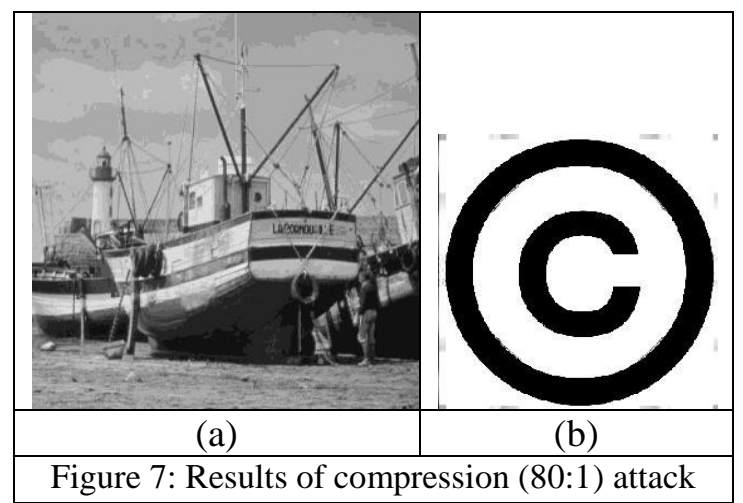

This algorithm also tested for rotation, cropping, and resizing attacks. For rotation, results for $50^{\circ}$ are shown in figure 8 . Cropping is very frequently used action on images in figure 9 , results for cropping has shown when only $25 \%$ area is remaining.

For resizing in fig 10, first the size of image is reduced to $128 \times 128$ and again carried back to original size $512 \times 512$. Simultaneously histogram equalization, sharpening and contrast adjustment attacks are performed. For histogram equalization the result has showed in figure 11. For sharpening attack, the sharpness of watermarked host image is increased by a factor 80 and the result has showed in figure 12. For contrast adjustment, the contrast of watermarked host image is decreased by $50 \%$ and the result has shown in fig 13 . Another important attack was image distort. A distort operation wrapping was performed on watermarked image and the corresponding images have shown in fig 14.

To verify the presence of watermark the correlation coefficient between original and extracted watermarks is given by the equation 10 and results are tabulated in table 1 .

The proposed algorithm results also compared with other existing algorithms. Those have been shown in table- 2 and table-3.

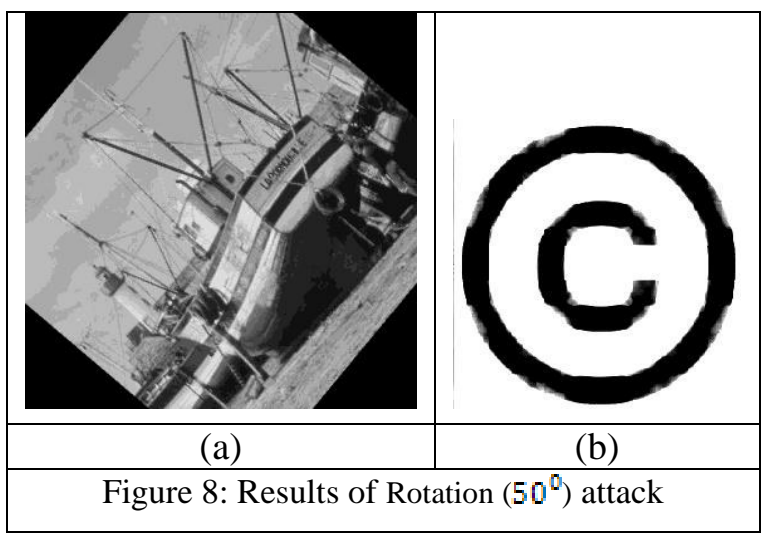

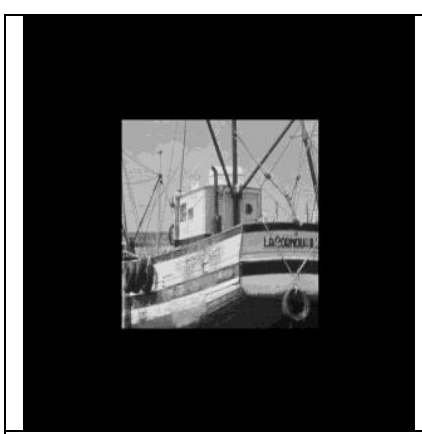

(a)
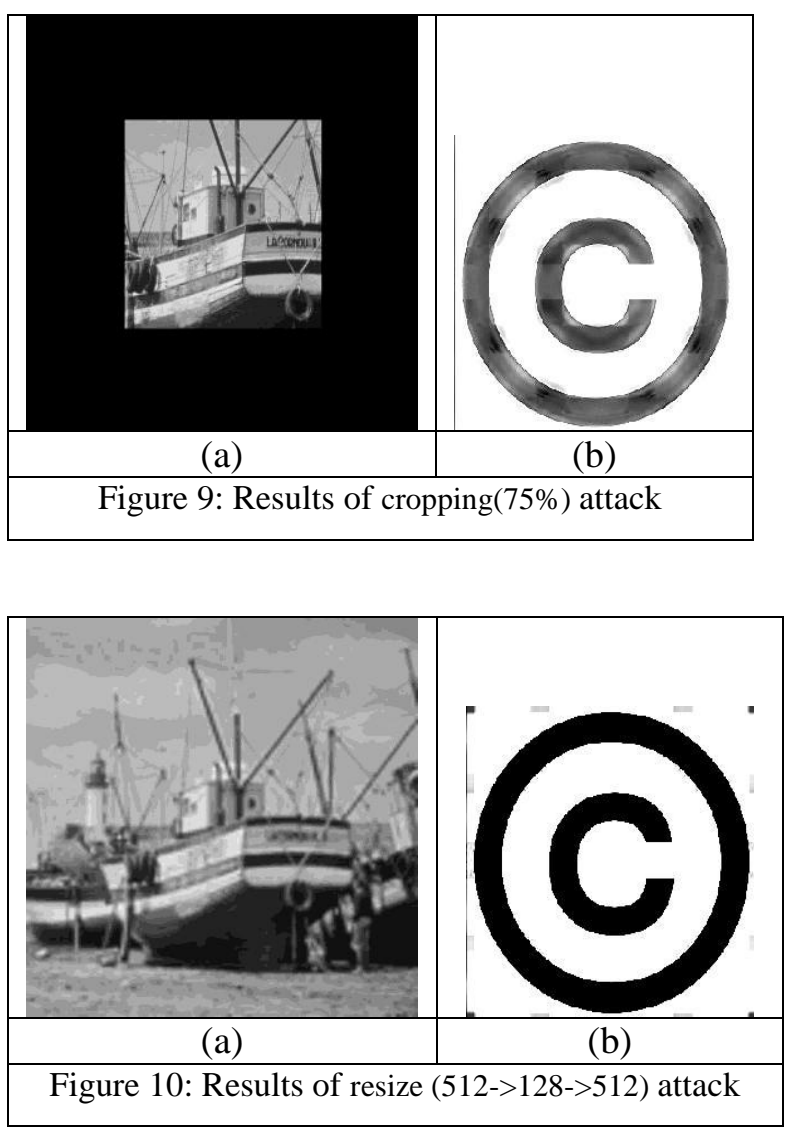

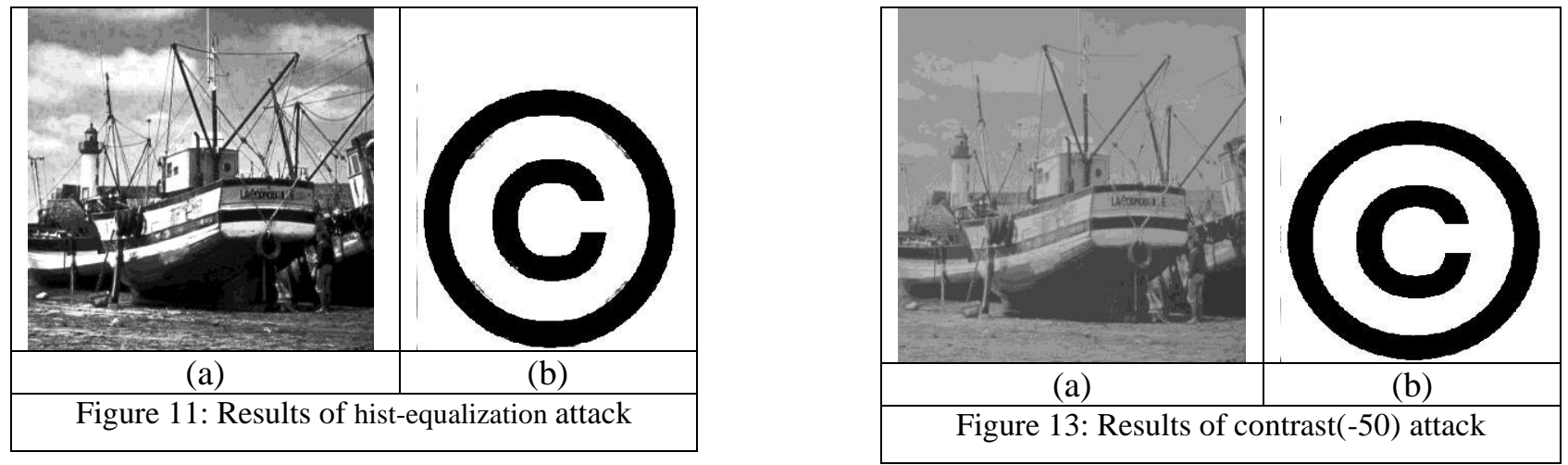

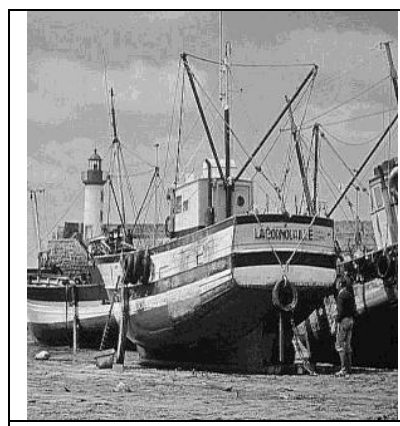

(a)

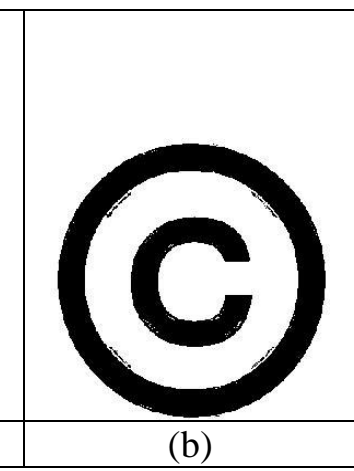

(b)

Figure 12: Results of sharpen(80) attack

\begin{tabular}{|c|c|c|c|c|c|c|}
\hline \multicolumn{7}{|c|}{ Table -1 : Comparison of NCC values } \\
\hline \multirow{2}{*}{ Attacks } & \multicolumn{2}{|c|}{$\begin{array}{l}\text { Existing method } \\
\text { FrFt-SVD (ref 42) }\end{array}$} & \multicolumn{2}{|c|}{$\begin{array}{l}\text { Existing method } \\
\text { DCT-SVD (ref 41) }\end{array}$} & \multicolumn{2}{|c|}{$\begin{array}{c}\text { Proposed method } \\
\text { DCT-SVD }\end{array}$} \\
\hline & Boat & Mandrill & Boat & Mandrill & Boat & Mandrill \\
\hline Average Filtering $(13 \times 13)$ & -0.3163 & -0.1281 & -0.1603 & -0.2066 & 0.9893 & 0.9871 \\
\hline Median Filtering (13 x 13) & -0.3158 & -0.1016 & 0.0184 & -0.1318 & 0.9868 & 0.9857 \\
\hline $\begin{array}{l}\text { Additive Gaussian } \\
\text { noise }(75 \%)\end{array}$ & 0.1833 & 0.1656 & 0.2514 & 0.3469 & 0.8949 & 0.8882 \\
\hline JPEG compression (80:1) & 0.8929 & 0.7865 & 0.9119 & 0.5648 & 0.9924 & 0.9680 \\
\hline Rotation $\left(50^{\circ}\right)$ & -0.5470 & -0.3626 & 0.3702 & 0.2404 & 0.9395 & 0.8351 \\
\hline $\begin{array}{l}\text { Cropping (25\% area } \\
\text { remaining) }\end{array}$ & -0.1232 & -0.1704 & 0.1735 & 0.0127 & 0.9688 & 0.9518 \\
\hline Resizing (512 -> 128 -> 512) & 0.4897 & 0.0560 & -0.1036 & -0.1880 & 0.9925 & 0.9859 \\
\hline Histogram equalization & 0.6009 & 0.7032 & 0.6932 & 0.6499 & 0.9874 & 0.9197 \\
\hline Sharpening (by factor 80 ) & 0.1607 & 0.1881 & 0.2758 & 0.2955 & 0.9811 & 0.9229 \\
\hline Wrapping & 0.2385 & 0.5486 & 0.4719 & 0.2306 & 0.9893 & 0.9722 \\
\hline Contrast adjustment(-50) & -0.7616 & -0.5952 & 0.6554 & -0.7272 & 0.9909 & 0.9776 \\
\hline
\end{tabular}




\begin{tabular}{|c|c|c|}
\hline \multicolumn{3}{|c|}{ Table -2 : Comparison of NCC values } \\
\hline Attacks & $\begin{array}{c}\text { Existing Method } \\
\text { (ref 37) } \\
\text { DCT-SVD }\end{array}$ & $\begin{array}{c}\text { Proposed } \\
\text { method } \\
\text { DCT-SVD }\end{array}$ \\
\hline $\begin{array}{c}\text { Gaussian Blur(5 } \\
\text { x 5) }\end{array}$ & 0.9894 & $\mathbf{0 . 9 9 1 7}$ \\
\hline $\begin{array}{c}\text { Gaussian Noise } \\
\text { (0.3) }\end{array}$ & 0.9942 & 0.9093 \\
\hline $\begin{array}{c}\text { Pixelate-2 } \\
\text { JPEG } \\
\text { compression } \\
\text { (30:1) }\end{array}$ & 0.9939 & $\mathbf{0 . 9 9 4 7}$ \\
\hline $\begin{array}{c}\text { Sharpening (by } \\
\text { factor 80) }\end{array}$ & 0.9998 & 0.9910 \\
\hline $\begin{array}{c}\text { Resizing (512 -> } \\
\text { 256 -> 512) }\end{array}$ & 0.9957 & 0.9937 \\
\hline $\begin{array}{c}\text { Rotation (20 } \\
0\end{array}$ & 0.7617 & $\mathbf{0 . 9 7 2 3}$ \\
\hline $\begin{array}{c}\text { Cropping (25\% } \\
\text { area remaining) }\end{array}$ & 0.9990 & 0.9183 \\
\hline $\begin{array}{c}\text { Contrast } \\
\text { adjustment(-20) }\end{array}$ & 0.9941 & $\mathbf{0 . 9 9 4 3}$ \\
\hline $\begin{array}{c}\text { Histogram } \\
\text { equalization }\end{array}$ & 9148 & $\mathbf{0 . 9 8 7 4}$ \\
\hline $\begin{array}{c}\text { Gamma } \\
\text { Correction (0.6) }\end{array}$ & 0.9993 & $\mathbf{0 . 9 9 9 5}$ \\
\hline
\end{tabular}

\begin{tabular}{|c|c|c|}
\hline Rotation-60 & ------ & $\mathbf{0 . 9 6 5 1}$ \\
\hline Rotation-90 & & \\
\hline & ----- & $\mathbf{0 . 9 9 2 1}$ \\
\hline
\end{tabular}

\section{CONCLUSIONS}

A new semi-blind reference watermarking scheme based on DCT and SVD is presented in which the watermark is visually meaningful gray scale image instead of a noisy-type Gaussian sequence. For the extraction of watermark a reliable watermark extraction scheme is constructed. Robustness of proposed method is carried out by a variety of attacks. The proposed algorithm results were compared with existing papers [41, 42]. No one can extract watermark without knowing the value of $\beta$. Hence the security is increased. The security of proposed method lies in the original reference image since no attacker can extract the data without access the original reference image. The robustness of proposed method was also compared with other existing algorithms [37, 40]. When comparing results with ref paper [37], the proposed results are superior in Gaussian blur, pixilated, sharpening, rotation, contrast adjustment, histogram equalization and gamma correction. But other attacks the existing algorithm was superior to proposed algorithm.

\section{REFERENCES}

[1] C.Rey and J.LDugelay, "A Survey of watermarking algorithms for image authentication," EURASIP Journal on Applied Signal Processing, Vol.6,pp.613-621,2002.

[2] V.Potdar, S. Han, and E. Chang, 2005. "A Survey of Digital Image Watermarking Techniques", in Proceedings of the 2005 IEEE International Conference on Industrial Informatics, pp. 709-716.

[3] P. Meerwald and A. Uhl, "A Survey of Waveletodomain watermarking Algorithms," Proceedings of the SPICE, Electronic Imaging, Security and Watermarking of Multimedia Contents III, pp.505 - 516,2001.

[4] M. Vidyasagar, S. Han and E. Chang, "A Survey of Digital Image Watermarking Techniques," $3^{\text {rd }}$ IEEE International Conference on Industrial Informatics (INDIN), Perth, Australia, pp.709-716, Aug 10 -12, 2005.

[5] Mr.Manjunatha Prasad.R,Dr.Shivaprakash Koliwad "A Comprehensive Survey of Contemporary Researches in Watermarking for Copyright Protection of Digital Images" IJCSNS International Journal of Computer Science and Network Security, VOL.9 No.4, pp. 91107,April 2009.

[6] N.Johnson and S. Katezenbeisser, "A Survey of Steganography Techniques," Chapter3, Techniques for Steganography and Digital Watermarking, Eds. Northwood, MA: Artech House, pp.43-75, Dec.1999.

[7] I. J. Cox, M. L. Miller, J. A. Bloom, Digital Watermarking, Academic Press,2002.

[8] F. Hartung and M. Kutter, "Multimedia Watermarking Techniques," in Proceedings of the IEEE, vol. 87, no.7, pp. 1079-1107, July 1999.

[9] I. Cox, M. Miller, J. Bloom, J. Fridrich, and T. Kalker, Digital Watermarking and Steganography, 2nd Ed. (The Morgan Kaufmann Series in Multimedia Information and Systems), 2nd ed. Morgan Kaufmann, 112007. 
[10] M. Barni and F. Bartolini, Watermarking Systems Engineering: Enabling Digital Assets Security and Other Applications. Imprint unknown, 12004.

[11] Arnold M., Schmucker M., Wolthusen S.D., "Techniques and application of Digital Watermarking and Content Protection", Eds. Northwood, Artech House, 2003.

[12] F. Cayre, C. Fontaine, and T. Furon, "Watermarking security: theory and practice," Signal Processing, IEEE Transactions on, vol. 53, no. 10, pp. 3976-3987, Oct. 2005.

[13] Chi-Kwong Chan , L.M. Cheng "Hiding data in images by simple LSB substitution "Pattern

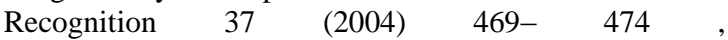
ww.elsevier.com/locate/patcog.

[14] Dr.M.A.Dorairangaswamy "A Robust Blind Image Watermarking Scheme in Spatial Domain for Copyright Protection" International Journal of Engineering and Technology Vol. 1, No.3,August, 2009 , ISSN: 17938236

[15] Piva A., Barni M., Bartolini F., Cappellini V."DCTbased watermark recovering without resorting to the uncorrupted original image", Proc. IEEE Int. Conf. on Image Processing, ICIP 97, Santa Barbara, CA, pp. 520-527, Oct. 1997.

[16] Podilchuk C., Zeng W., "Perceptual watermarking of still images", Proc. IEEE 1st Workshop on Multimedia Signal Processing, Princeton, NJ, pp. 363368, June 23-25, 1997.

[17] Tao B., Dickinson B., "Adaptive watermarking in the DCT domain", IEEE Int. Conf. on Acoustics, Speech and Signal Processing, pp. 20-46, Apr.1997.

[18] Wolfgang R.B., Podilchuk C.I., Delp E.J., "Perceptual watermarks for digital images and video", Proc. IEEE, vol. 87, pp. 1108-1126, July 1999.

[19] Hernández J.R., Amado M., Gonzalez F.P., "DCTDomain watermarking techniques for still images: Detector performance analysis and a new structure ", IEEE Transactions of Image Processing, vol. 9, pp. 55-68, Jan. 2000.

[20] Gu F., Lu Z.M., Pan J.S., "Multipurpose image watermarking in DCT domain using subsampling", IEEE Int. Symposium On Circuits and Systems, vol. 5, pp. 4417- 4420, May 2005.

[21] Li X., Xue X. "Improved robust watermarking in DCT domain for color images", Proc. IEEE Int. Conf. On Advanced Information Networking and Applications, IEEE-AINA 2004, vol. 1, pp. 53 - 58, 2004.

[22] Fanman Meng, Hong Peng, Zheng, Pei,Jun, Wang, “ A novel blind image watermarking scheme based on support vector machine in DCT domain," in IEEE International Conference On Computational Intelligence and Security,2008.

[23] C.S.Shieh, H.C.Huang, F.H.Wang and J.S.Pan, "Genetic watermarking based on transform domain technique," Pattern Recognition,37(3):555-565, March 2004.

[24] Zha0 Rui-mei, Lian Hua, Pang Hua-wei and Hu Bo-ning, "A Blind Watermarking Algorithm Based on DCT," Second International Symposium on Intelligent
Information Technology Application, vol.3, pp.821824,2008 .

[25] Zha0 Rui-mei, Lian Hua, Pang Hua-wei and Hu Bo-ning, "A Watermarking Algorithm by Modifying AC Coefficients in DCT domain," International Symposium on Information Science and engineering, vol.2, pp.159162,2008 .

[26] S. D. Lin and C.-F. Chen,"A Robust DCT-Based Watermarking for Copyright Protection," IEEE Transactions on Consumer Electronics, 46(3), August 2000, pp. 415-421.

[27] Gengming Zhu, and Nong Sang "Watermarking Algorithm Research and Implementation Based on DCT Block " proceedings of world academy of science, engineering and technology volume 35 november 2008 issn $2070-3740$

[28] Ahmidi N. Safa R., "A Novel DCT-based Approach for Secure Color Image Watermarking", Proc. Int. Conf. on Information Technology: Coding and Computing (ITCC'04), page 709, IEEE, 2004.

[29] Chu W.C., "DCT-based image watermarking using subsampling", IEEE Transactions on Multimedia, vol. 5, no. 1, pp. 34-38, June 2005.

[30] LIU Rui-zhen, Tan Tie-niu.2001. "SVD Based Digital Watermarking Method". Acta Electronica Sinica, Vol.29, No.2.

[31] R.Z. Liu and T.N. Tan,2002. "An SVD-Based Watermarking Scheme for Protecting Rightful Ownership", IEEE Trans. On Multimedia, Vol. 4, No. 1, pp. 121-128.

[32] J. M. Shieh, D. C. Lou, and M. C. Chang, "A semi-blind watermarking scheme based on singular value decomposition", computer standards \& interface 28 (2006) 428-440.

[33] Aslantas, A singular-value Decomposition-Based Image Watermarking Using Genetic Algorithm.In: International Journal of Electronics and Communications vol.62, (2007), pp.386-393.

[34] C.C. Lai, H.C. Huang and C.C. Tsai, Image Watermarking Scheme Using Singular Value Decomposition and Micro-genetic Algorithm. In: Proceedings of International Conference on Intelligent Information Hiding and Multimedia Signal Processing, (2008), pp.469-472.

[35] X. Qi, S. Bialkowski and G. Brimley, An Adaptive QIMand SVD-based Digital Image Watermarking Scheme in The Wavelet Domain. In: Proceedings of IEEE International Conference on Image Processing, (2008), pp.421-424.

[36] B.Chandra Mohan, , S.Srinivaskumar, B.N.Chatterji, “A Robust Digital Image Watermarking Scheme using Singular Value Decomposition (SVD), Dither Quantization and Edge Detection," ICGST-GVIP Journal, ISSN: 1687-398X , Volume 8, Issue 1, June 2008.

[37] Alexander Sverdlov, Scott Dexter, Ahmet M. Eskicioglu "Robust SVD DCT based watermarking for copyright protection", IEEE Transactions on Image Processing, 10(5), May 2001, pp. 724-735. 
[38] Liu Quan, and AI Qingsong “A combination of DCT based and SVD based watermarking," ICSP proceedings of IEEE International conference on signal processing, pp. 873-876, 2004.

[39] Fangjun Huang and Zhi-Hong Guan, A hybrid SVDDCT watermarking method based on LPSNR, Pattern Recognition Letters, vol. 25, pp. 1769--1775, 2004

[40] Feng Liu, and Yangguang Liu, "A Watermarking Algorithm for Digital Image Based on DCT and SVD,"Congress on Image and Signal Processing, pp. 380-383, 2008

[41] Satyanarayana Murty .P, P. Rajesh Kumar, "A Semi Blind Watermarking Scheme Using DCT and SVD", $1^{\text {st }}$ International Conference On Emerging trends in signal processing and VLSI design,2010

[42] Gaurav Bahtnagar and Bala Subramanian"Reference Watermarking Scheme in Fractional Fourier Transform Domain Using Singular Value Decomposition "International journal of information Processing,2(4), pp.88-97,2008.

\section{AUTHOR'S PROFILE}

P. Satyanarayana Murty is currently working as Sr.Associate Professor in ECE Department, GIITS, Engineering College, Vishakapatnam, and Andhra Pradesh, India. He is working towards his Ph.D.at AU College of Engineering, Vishakapatnam, India. He received his M.Tech from Jawaharlal Nehru Technological University, Hyderabad, India. He has fifteen years experience of teaching undergraduate students and post graduate students. His research interests are in the areas of image watermarking, and image compression

Dr. P. Rajesh Kumar is currently Associate Professor in ECE Department, AU College of Engineering, Vishakapatnam, India. $\mathrm{He}$ received his M.Tech and Ph.D. from AndhraUniversity, Vishakapatnam, India. He has eleven years experience of teaching undergraduate and postgraduate students and guided number of post-graduate theses. He has published 10 research papers in National and International journals. Presently he is guiding six Ph.D students in the area of digital signal processing and Image processing. His research interests are in the areas of digital image processing and digital signal processing. 